The bluefin tuna from southern and lower California are essentially one species and, until adequate descriptions are available from all localities, must be assigned to the same species as Thunnus thynnus of the Atlantic. Lacking material from Japan, comparison with Kishinouye's (1923) description of Thunnus orientalis (the oriental bluefin) shows that this is probably a different species.

With regard to the chief morphological features, it was found that the appearance of the viscera in situ is a valuable identifying character. Other differences appear in the presence or absence of air bladder, differences in excretory system, and the circulatory system which is unique in many respects. The differences in circulatory system were used by Kishinouye in classifying the tunas. The present observations were confined largely, but not exclusively, to the arterial system. The post-cardinal vein is present in all but the genus Thunnus, but its course and degree of development differ. The skeletal elements are remarkably alike, and the authors believe that they offer less promise for specific identification than other anatomical parts. Nevertheless a key is given to the five species studied, based on these skeletal elements; also a table giving a summary of anatomical differences.

In the most important characters the skipjack differs from all the others. Alone it can be distinguished at once by the external features. It lacks an air bladder. The intestine is not folded. The ventral view of the viscera in situ is distinctive and differentiates it. In its blood system and excretory system it differs sharply from the others. It is the easiest to identify of the five species.

Foreseeing the necessity of a more detailed population study of some species, a large number of both external and internal measurements and counts were added to the routine. These are reserved for a future work.

\section{A METEORIC THEORY OF THE ORIGIN OF THE EARTH AND PLANETS}

$\mathrm{O}$ J. SCHMIDT has a paper with this title in Comptes Rendus (Doklady) de L'Académie des Sciences de L'URSS. (45, No. $6 ; 1944)$, in which he propounds a new theory of the origin of the planetary system. At the basis of the theory there are two fundamental facts--the rotation of the Galaxy and the presence near its central plane of large masses of obscuring matter. During its motion round the centre of gravity of the Galaxy, the sun crossed a dark cloud of dust and meteorites and captured portions of this, compelling the particles to revolve around its centre. In the course of time these captured particles united into larger formations, thus producing the planets.

In a previous paper (C.R. Acad. Sci. URSS., 44, No. $1 ; 1944)$, the author dealt with the formation of binaries in a rotating Galaxy. The motions of stars are a combined result of the attraction of the central galactic masses and of external masses and the neighbouring individual stars, and under certain conditions two stars can come into such a position that they are drawn closer together, so that capture and the formation of a double star occur. The following assumptions, considered to correspond to statistical averages, were made in dealing with this problem : (a) The stars move along circular orbits, subject to the attraction of the Galaxy, supposed to be concentrated in its centre. (b) The orbits lie in different planes inclined at small angles to one another. (c) Capture takes place when the two stars pass the position of the shortest distance between the orbits.

On these assumptions, a formula has been derived which connects the semi-major axis and eccentricity of the orbit of the binary with a quantity which depends on "the galactic parameter of the stars". This is a function of the distance to the galactic centre, the mass of the inner portion of the Galaxy, the total mass of the two stars, and the angle between the orbital planes of the stars previous to capture. While the formula is not strictly applicable in every case, as it considers mean results, nevertheless it is sufficiently accurate to apply to the problem of the capture of meteoric matter by the sun.

It is essential to the theory that the plane of the sun's galactic orbit should be inclined at an angle of about $3^{\circ}$ to the central plane of the Galaxy. If the sun while at one of the nodes of its orbit passes through a cloud of matter, meteorites are captured in accordance with the same laws as underlie the formation of double stars. Both direct and retrograde motions take place with the captured meteorites; but if the sun passes near the edge of the cloud its density is not uniform, and in consequence more meteorites revolve in one direction about the sun. Those revolving in the opposite direction collide with the more numerous portion of the swarm and lose their momentum, ultimately falling into the sun. The main mass segregates in the course of time into larger bodies from which the planets are formed, and this segregation proceeds through smaller particles falling on larger ones just as meteorites fall on the earth at present. As the swarm of meteorites has the form of a flat lens, the planets in the process of formation have their orbits lying approximately in one plane - the central plane of the lens-and as the meteorites ultimately revolve in one direction, for reasons already suggested, the planets will also revolve in one direction. In the early stage the major axes of the swarm have a tendency to distribute their axes along the shortest distance between the galactic orbit of the sun and that of the cloud, but they are deflected from this direction by mutual perturbations. Hence no planet formed in the manner suggested can have a preferential direction for its major axis.

The problem of the angular momenta of the planets, which has been an insuperable difficulty with many theories of the formation of the solar system, is avoided by the present view, as the galactic momentum of the meteorites supplies the necessary angular momenta. Dr. Schmidt hopes that the theory will be further developed to bring within its scope such problems as the rotation of the sun and of the planets, the age of the planetary system, the formation of the satellites, etc. In addition, certain features concerning the internal structure of the earth are explicable, and Dr. Schmidt is engaged in the preparation of papers which will discuss such problems. The present paper contains some quantitative results regarding the distribution of mass and momentum in the planetary system and also the extent of agreement between the theory and observational evidence. These are largely provisional, and additional evidence in support of the theory will be forthcoming in the papers which are to be published in due course. 